\title{
Electronic Density of States of Atomically Resolved Single-Walled Carbon Nanotubes: Van Hove Singularities and End States
}

\section{Citation}

Kim, Philip, Teri W. Odom, Jin-Lin Huang, and Charles M. Lieber. 1999. "Electronic Density of States of Atomically Resolved Single-Walled Carbon Nanotubes: Van Hove Singularities and End States." Physical Review Letters 82 (6): 1225-28. https://doi.org/10.1103/physrevlett.82.1225.

\section{Permanent link}

http://nrs.harvard.edu/urn-3:HUL.InstRepos:41417274

\section{Terms of Use}

This article was downloaded from Harvard University's DASH repository, and is made available under the terms and conditions applicable to Other Posted Material, as set forth at http:// nrs.harvard.edu/urn-3:HUL.InstRepos:dash.current.terms-of-use\#LAA

\section{Share Your Story}

The Harvard community has made this article openly available. Please share how this access benefits you. Submit a story. 


\title{
Electronic Density of States of Atomically Resolved Single-Walled Carbon Nanotubes: Van Hove Singularities and End States
}

\author{
Philip Kim, Teri W. Odom, Jin-Lin Huang, and Charles M. Lieber \\ Harvard University, 12 Oxford Street, Cambridge, MA 02138
}

\begin{abstract}
The electronic density of states of atomically resolved single-walled carbon nanotubes have been investigated using scanning tunneling microscopy. Peaks in the density of states due to the onedimensional nanotube band structure have been characterized and compared with the results of tight-binding calculations. In addition, tunneling spectroscopy measurements recorded along the axis of an atomically-resolved nanotube exhibit new, low-energy peaks in the density of states near the tube end. Calculations suggest that these features arise from the specific arrangement of carbon atoms that close the nanotube end.
\end{abstract}

PACS numbers:71.20.Tx;61.16.Ch;71.20.-b;73.20.At

The electronic properties of single-walled carbon nanotubes (SWNTs) are currently the focus of considerable interest [1]. According to theory [2] 1 , SWNTs can exhibit either metallic or semiconducting behavior depending on diameter and helicity. Recent scanning tunneling microscopy (STM) studies of SWNT [5,6] have confirmed this predicted behavior, and have reported peaks in the density of states (DOS), Van Hove singularities (VHS), that are believed to reflect the 1D band structure of the SWNTs. A detailed experimental comparison with theory has not been carried out, although such a comparison is critical for advancing our understaning of these fascinating materials. For example, chiral SWNTs have unit cells that can be significantly larger than the cells of achiral SWNTs of similar diameter, and thus chiral tubes may exhibit a larger number of VHS than achiral ones [7]. Recent theoretical work [8.9] suggested, however, that semiconducting (or metallic) SWNTs of similar diameters will have a similar number of VHS near the Fermi level, independent of chiral angle. In addition, the electronic properties of localized SWNT structures, including end caps, junctions and bends [10 13], which are essential to proposed device applications, have not been characterized experimentally in atomically resolved structures.

In this Letter, we report STM investigations of the electronic structure of atomically resolved SWNTs and compare these results with tight-binding calculations. Significantly, we find that the VHS in the DOS calculated using a straight-forward zone-folding approach agree with the major features observed in our experiments. We have observed new peaks in the local DOS (LDOS) at an end of a metallic SWNT and compared these results to calculations. This analysis suggests that the new peaks can be associated with a specific topology required to cap the SWNT. The implications of these results and important unresolved issues are discussed.

Experimental procedures and are described elsewhere in detail 66,14. In brief, SWNT samples were prepared by laser vaporization [15], purified and then deposited onto a $\mathrm{Au}(111) /$ mica substrate. Immediately after deposition, the sample was loaded into a UHV STM that was stabilized at $77 \mathrm{~K}$; all of the experimental data reported in this Letter was recorded at $77 \mathrm{~K}$. Imaging and spectroscopy were measured using etched tungsten tips with the bias $(V)$ applied to the tip. Spectroscopy measurements were made by recording and averaging 5 to 10 tunneling current $(I)$ versus $V(I-V)$ curves at specific locations on atomically resolved SWNTs. The feedback loop was open during the $I-V$ measurement while the setpoint was the same as that of imaging. The tunneling conductance, $d I / d V$, was obtained by numerical differentiation.
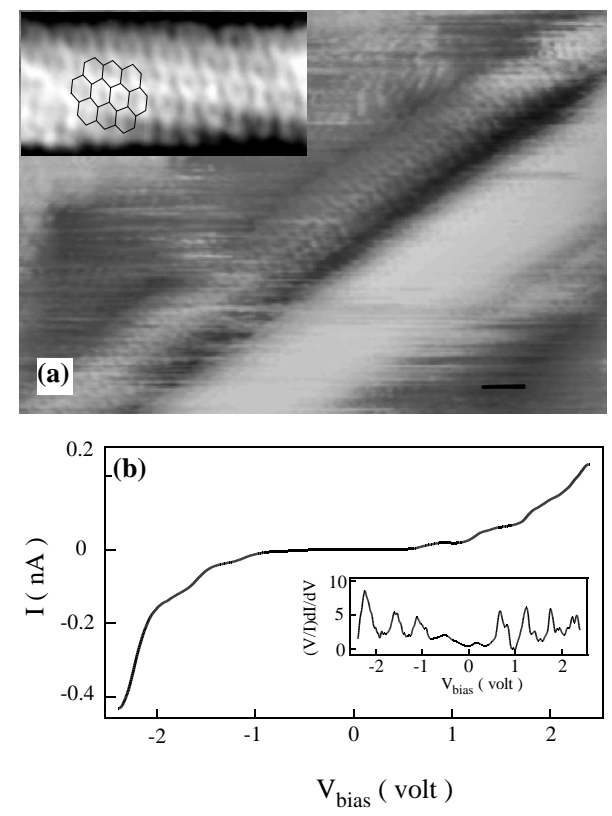

FIG. 1. (a) STM image of SWNTs recorded at $I=0.12$ $\mathrm{nA}$ and $V=550 \mathrm{mV}$. Tunneling spectra were taken on the isolated upper tube. The black scale bar is $1 \mathrm{~nm}$. The inset shows an atomic resolution image of this tube. A portion of a hexagonal lattice is overlaid to guide the eye. (b) $I-V$ data recorded on the SWNT in (a). The inset shows the normalized conductance, $(d I / d V /(I / V))$. 
An atomically resolved STM image of several SWNTs is shown in Fig. 1(a). The upper isolated SWNT rests on the Au surface and is on the edge of a small rope that contains around 10 nanotubes. Below we concentrate our analysis on this individual SWNT. The diameter and chiral angle measured for this tube were $1.35 \pm$ $0.1 \mathrm{~nm}$ and $-20 \pm 1^{\circ}$, respectively. These values are consistent with $(13,7)$ and $(14,7)$ indices [6, 14, where $(13,7)$ and $(14,7)$ are expected to be metallic and semiconducting respectively. The $I-V$ data exhibits metallic behavior with relatively sharp, stepwise increases at larger $|V|$ (Fig. 1(b)). The $I-V$ curves have a finite slope, and thus the normalized conductance $(V / I)(d I / d V)$, which is proportional to the LDOS, has appreciable non-zero value around $V=0$ as expected for a metal (Fig. 11(b) inset). This suggests that the $(13,7)$ indices are the best description of the tube (we address this point further below). At larger $|V|$, several sharp peaks are clearly seen in $d I / d V$ and $(V / I)(d I / d V)$ vs, $V$. These peaks were observed in four independent data sets recorded at different positions along this atomically-resolved tube (but not on the $\mathrm{Au}(111)$ substrate), and thus we believe these reproducible features are intrinsic to the SWNT. We attribute these peaks to the VHS resulting from the extremal points in the 1D energy bands [16].

The availability of spectroscopic data for atomicallyresolved nanotubes represents a unique opportunity for comparison with theory. In this regard, we have calculated the band structure of a $(13,7)$ SWNT using the tight-binding method. If only $\pi$ and $\pi^{*}$ orbitals are considered, the SWNT band structure can be constructed by zone-folding the $2 \mathrm{D}$ graphene band structure into the $1 \mathrm{D}$ Brillouin zone specified by the $(n, m)$ indices [1]. Fig. 2(a) shows the graphene $\pi$ band structure around the corner point $(\mathbf{K})$ of the hexagonal Brillouin zone. For the metallic $(13,7)$ tube, the degenerate $1 \mathrm{D}$ bands which cross $\mathbf{K}$ result in a finite DOS at the Fermi level. Note that the energy dispersion is isotropic (circular contours) near $\mathbf{K}$, and becomes anisotropic (rounded triangular contours) away from $\mathbf{K}$. Therefore, the first two VHS in the 1D bands closest to $\mathbf{K}$ (depicted by $\boldsymbol{\Lambda}, \mathbf{v}$ ) have a smaller splitting in the energy due to the small anisotropy around $\mathrm{K}$, while the next two VHS (depicted by $\mathbf{\square}, \mathbf{\text { ) have a }}$ larger splitting due to increasing anisotropy. If the energy dispersion were completely isotropic, both sets of peaks would be degenerate. Values for the hopping integral, $V_{p p \pi}$, reported in the literature range from ca. 2.4 to $2.9 \mathrm{eV}$ [1.2, 6.5,8, 17]. We used a value of $2.5 \mathrm{eV}$ determined from previous measurements of the energy gap vs. diameter $[6]$.

Our STS data shows relatively good agreement with the DOS for a $(13,7)$ tube calculated using the zonefolding approach (Fig. 2(b)). The agreement between the VHS positions determined from our $d I / d V$ data and calculations are especially good below the Fermi energy $\left(E_{F}\right)$ where the first seven peaks correspond well. De- viations between experiment data and calculations are larger above than below $E_{F}$. The observed differences may be due to the band repulsion, which arises from the curvature-induced hybridization, or surface-tube interaction that were not accounted for in our calculations. Detailed $a b$ initio calculation [18] have shown that the effect of curvature induced by hybridization is much higher in $\pi^{*} / \sigma^{*}$ than $\pi / \sigma$ orbitals. Bands above $E_{F}$ are thus more susceptible to the hybridization effect, and this could explain the greater deviations between experiments and calculation that we observe for the empty states. In the future, we believe that comparison between experiment and more detailed calculations should help (a) to resolve such subtle but important points, and (b) to understand how inter-tube and tube-substrate interactions affect SWNT band structure.
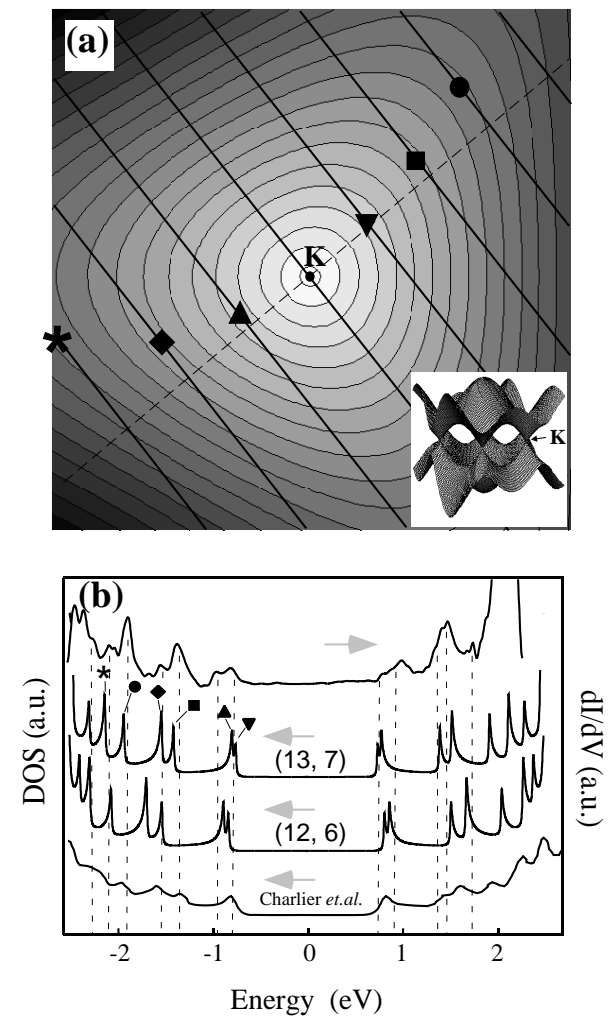

FIG. 2. (a) Energy dispersion of the $\pi$-band of graphene sheet near the $\mathbf{K}$. The solid lines correspond to $(13,7) 1 \mathrm{D}$ bands obtained by the zone-folding. Symbols are located at the positions where VHS occur in these 1D bands. The inset depicts a three dimensional view of graphene $\pi / \pi^{*}$ bands. (b) Comparison of DOS obtained from our experiment (upper curve) and $\pi$-only tight binding calculation for $(13,7)$ SWNT (second curve from top). The broken vertical lines indicate the positions of VHS in the tunneling spectra after consideration of thermal broadening convolution. The symbols correspond to the VHS shown in (a). The calculated DOS of $(12,6)$ and an independent calculation for a $(13,7)$ tube $[9]$ are included for comparison. 

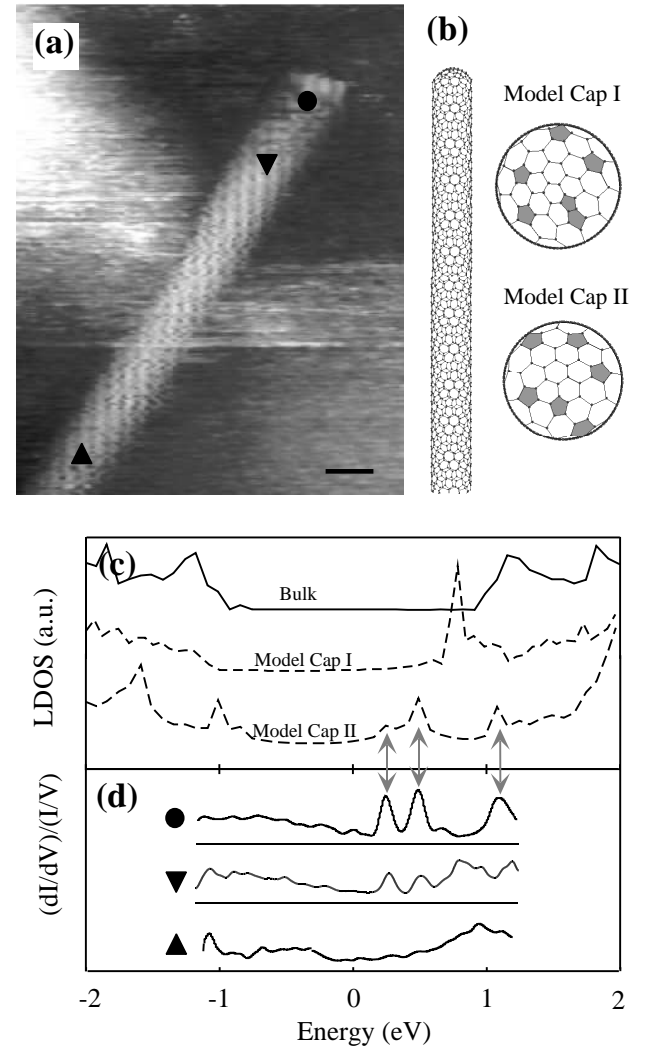

FIG. 3. (a) STM image of the end of a SWNT with $I=0.15 \mathrm{nA}$ and $V=750 \mathrm{mV}$. The scale bar is $1 \mathrm{~nm}$, and symbols $(\bullet, \boldsymbol{\nabla} \& \mathbf{\Lambda})$ correspond to the locations where the tunneling spectra in (d) were recorded. (b) A model $(13,-2)$ SWNT with two different cap structures; the pentagons in the caps are shaded gray. (c) LDOS obtained from a tight-binding calculation on capped $(13,-2)$ tubes. The solid and dashed curves correspond to the calculated bulk DOS and end DOS of cap I and cap II, respectively. (d) Experimental tunneling spectra from the end $(\bullet)$, near the end $(\boldsymbol{\nabla})$, and far from end (४). Similar features in $\bullet, \nabla$ and cap II are highlighted by gray arrows.

In addition, we have compared these results to a recent $\sigma+\pi$ calculation for a $(13,7)$ SWNT [9] and a $\pi$ only calculation for a closely related set of indices. The bottom curve shown in Fig. 2(b) is adopted from [9], and was obtained using $2 \mathrm{~s}$ and $2 \mathrm{p}$ orbitals. Although the direct comparison is difficult due to an large broadening, all peaks within $\pm 2 \mathrm{eV}$ match well with our $\pi$-only calculation. This comparison suggests that curvature induced hybridization is only a small perturbation within the experimental energy scale $(|V|<2 \mathrm{~V})$ for the $(13,7)$ tube. We have also investigated the sensitivity of the DOS to $(n, m)$ indices by calculating the DOS of the next closest metallic SWNT to our experimental diameter and angle;that is, a $(12,6)$ tube. Significantly, the calculated VHS for this $(12,6)$ tube deviate much more from the experimental DOS peaks than in the case of the $(13,7)$ tube(Fig. 2(b)). We believe that this analysis not only substantiates our assignment of the indices in Fig. 1](a), but more importantly, demonstrates the sensitivity of detailed DOS to subtle variations in diameter and chirality.

Lastly, we have also investigated the electronic structure of the ends of atomically-resolved SWNTs. Analogous to the resonant or localized surface states in bulk crystals 19], resonant or localized states are expected at the ends of nanotubes [10]. In accordance with Euler's rule, a capped end should contain six five-membered carbon rings (pentagons). The presence of these topological defects can cause dramatic changes in the LDOS near the end of a nanotube 20. Previous STM studies of multi-walled nanotubes 13 reported localized states at the ends of these tubes, although the atomic structure of the tubes was not resolved. To the best of our knowledge, STM studies of the ends of SWNT have not been reported. Fig. 3(a) shows an atomically-resolved image of the end of an isolated SWNT that has also been characterized spectroscopically. The rounded structure exhibited in this and bias-dependent images (e.g., insets Fig. 4(a)) suggests strongly that the end is closed, although the atomic structure cannot be obtained since the tube axis is parallel to the image plane. These images enable us to assign the nanotube $(13,-2)$ indices (the lefthanded counterpart to an $(11,2)$ tube). The expected metallic behavior of the $(13,-2)$ tube was confirmed in $(V / I)(d I / d V)$ data recorded away from the end ( $\boldsymbol{\Lambda}$ in Fig. 3(a),(d)). Significantly, spectroscopic data recorded at and close to the SWNT end $(\bullet \&$ in Fig. $3(d))$ show two distinct peaks at $250 \mathrm{mV}$ and $500 \mathrm{mV}$ that decay and eventually disappear in bulk DOS recorded far from the tube end $(\mathbf{\Lambda})$. The peaks were observed in 10 independent data sets recorded at the tube end, and are very reproducible.

To investigate the origin of these new features, we carried out tight-binding calculations for a $(13,-2)$ model tube with different end caps (Fig. 3(b)). All the models exhibit a bulk DOS far from the end (solid curve in Fig. 3(c)); however, near the end, the LDOS show pronounced differences from the bulk DOS:two or more peaks appear above the $E_{F}$, and these peaks decay upon moving away from the end to the bulk. (Fig. B(b)) shows two representative cap models. These models were chosen to illustrate the relatively large peak differences for caps closed with isolated vs. adjacent pentagons. These topological configurations are not unique, although additional calculations show that other isolated (adjacent) pentagon configuration have similar LDOS. Significantly, the LDOS obtained from the calculation for cap II shows excellent agreement with the measured LDOS at the tube end, while cap I does not (Fig. 3( (c-d)). The positions of the two end LDOS peaks as well as the first band edge of cap II match well with those from the experimental spectra.These results suggest that the topological arrangement of pentagons is responsible for the observed localized features in the experimental DOS at the SWNT end, and are thus similar to conclusions drawn 
from measurements on multi-walled nanotubes that were not atomically-resolved 13].

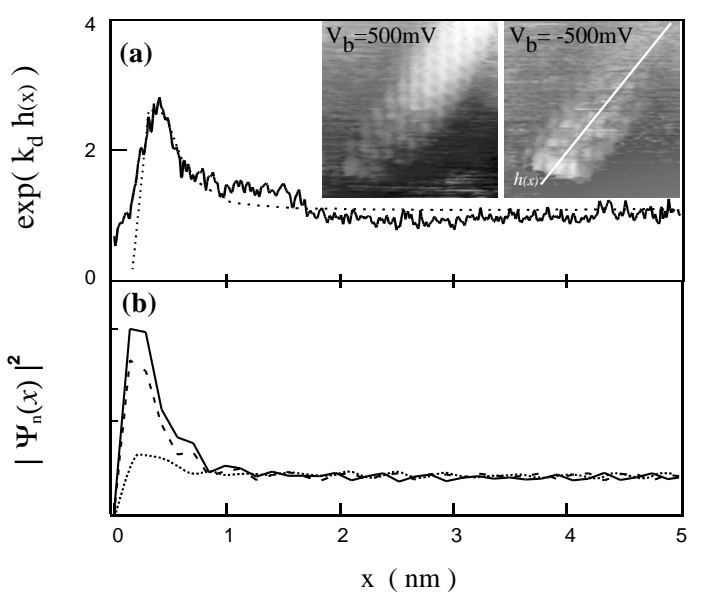

FIG. 4. (a) (inset) STM images recorded at different voltages on the SWNT end in Fig. 3. The white indicates the $h(x)$ cross section. The solid line in (a) corresponds to $\exp \left(k_{d} h(x)\right)$, where $k_{d}=2 \AA^{-1}$, and the dotted line is the integrated LDOS (0-500 meV) from our calculation. (b) The solid ,broken, and dotted lines depict wavefunction probability (arb. units), $\left|\psi_{n}(x)\right|^{2}$, of cap II as a function of position $x$ for eigenenergies of 500, 250, and $320 \mathrm{meV}$, respectively.

The nature of the DOS peaks at the nanotube end was further investigated using bias dependent STM imaging. At the bias of strong DOS peak, $-500 \mathrm{mV}$, the tip-nanotube separation $h(x)$ decays with increasing $x$, where $x$ is the distance from the tube end (inset, Fig. 4(a)). As indicated in Fig. 14(a), $\exp \left(k_{d} h(x)\right)$, which is proportional to the integrated LDOS [21], sharply increases around the capped end of the tube and then decays with a characteristic length scale ca. $1.2 \mathrm{~nm}$. Our tight-binding calculation suggests that this decay can be attributed to resonant end states. Wavefunctions whose eigenenergies correspond to the LDOS peaks (250, 500 $\mathrm{meV}$ ) decay exponentially from the end into the bulk but retain a finite magnitude (Fig. A(b)); this type of decay is a signature of a resonant state [19. Note that $h(x)$ does not decay at $V$ far from the resonance (e.g. Fig. 3(a)) nor do wavefunctions whose eigenenergies are away from the end LDOS peaks decay with $x$ (Fig. $4(\mathrm{~b})$ ). Resonant end states in metallic tubes could serve an important function in electronic devices by improving the contact between nanotubes and electrodes.

In summary, we have reported sharp VHS in the DOS of atomically-resolved SWNTs using STM, and have compared these data to tight-binding calculations for specific tube indices. A remarkably good agreement was obtained between experiment and $\pi$-only calculations, although deviations suggest that further work will be needed to understand fully the band structure of SWNTs in contact with surfaces. Pronounced peaks in the LDOS were also observed at the end of an atomically-resolved metallic SWNT. Comparison of these data with calculation suggests that the topological arrangement of pentagons is responsible for the localized features in the experiment. Such end states could be used to couple nanotube effectively to electrodes in future nanotube-based devices.

We thank R.E. Smalley (Rice) and C.-L. Cheung (Harvard) for SWNT samples. T.W.O. acknowledges predoctoral fellowship support from the NSF, and C.M.L. acknowledges support of this work by the National Science Foundation (DMR-9306684).

[1] M. S. Dresselhaus et al., Science of Fullerenes and Carbon Nanotubes (Academic, San Diego,1996).

[2] J. W. Mintmire et al., Phys. Rev. Lett. 68, 631 (1992).

[3] N. Hamada, et al., Phys. Rev. Lett. 68, 1579 (1992).

[4] R. Saito et al., Appl. Phys. Lett. 60, 2204 (1992).

[5] J. W. G. Wildoer et al., Nature 391, 59 (1998).

[6] T. W. Odom et al., Nature 391, 62 (1998).

[7] M. S. Dresselhaus, Nature 391, 19 (1998).

[8] C. T. White, and J. W. Mintmire, Nature 394, 29 (1998).

[9] J. -C. Charlier et al., Phys. Rev. B. 57, R15037 (1998).

[10] P.H. Lambin et al., J. Phys. Chem. Solid 58, 1833 (1997).

[11] L. Chico et. al., Phys. Rev. Lett. 76, 971 (1996).

[12] C. L. Kane et al., Phys. Rev. Lett. 78, 1932 (1997).

[13] D. L. Carroll et al., Phys. Rev. Lett. 78, 2811 (1997).

[14] T. W. Odom et al., J. Mater. Res. 13,2380 (1998)

[15] T. Guo et al., Chem. Phys. Lett. 243, 49 (1995).

[16] Broad features observed in $(V / I) / d I / d V$ near $V=0$ do not correspond to significant changes in the DOS; the variation in $d I / d V$ is small compared to that of the VHS occurring at the band edges. These small variations in the DOS could be attributed to the interaction between tube and Au substrate or adjacent tubes.

[17] C. T. White et al., Phys. Res. B. 47, 5485 (1993).

[18] X. Blase et al., Phys. Rev. Lett. 72, 1878 (1994).

[19] M. Lannoo, and P. Friedel, Atomic and Electronic Structure of Surfaces, (Springer-Verlag, Berlin, 1991).

[20] R. Tamura et al., Phys. Rev. B. 52, 6015 (1995).

[21] If the wave function normal to the tube axis decays exponentially with a constant inverse decay length $k_{d}$, $\int_{0}^{e V} d E \operatorname{LDOS}(x, E) \sim \exp \left(k_{d} h(x)\right) . k_{d}$ was obtained from experimental current versus distance measurements. 